\title{
Model and Controller Order Reduction for Infinite Dimensional Systems
}

\author{
Fatmawati $^{1{ }^{1 *}, \text { R. Saragih }}{ }^{1}$, B. Riyanto ${ }^{3} \&$ Y. Soeharyadi ${ }^{2}$ \\ ${ }^{1}$ Industrial and Financial Mathematics Group \\ email: fatma47@students.itb.ac.id; roberd@math.itb.ac.id \\ ${ }^{2}$ Analysis and Geometry Group \\ email: yudish@math.itb.ac.id \\ ${ }^{3}$ School of Electrical Engineering and Informatics \\ email: briyanto@1skk.ee.itb.ac.id \\ Institut Teknologi Bandung, Jalan Ganesa 10 Bandung, Indonesia.
}

\begin{abstract}
This paper presents a reduced order model problem using reciprocal transformation and balanced truncation followed by low order controller design of infinite dimensional systems. The class of systems considered is that of an exponentially stable state linear systems $(A, B, C)$, where operator $A$ has a bounded inverse, and the operator $B$ and $C$ are of finite-rank and bounded. We can connect the system $(A, B, C)$ with its reciprocal system via the solutions of the Lyapunov equations. The realization of the reciprocal system is reduced by balanced truncation. This result is further translated using reciprocal transformation as the reduced-order model for the systems $(A, B, C)$. Then the low order controller is designed based on the reduced order model. The numerical examples are studied using simulations of Euler-Bernoulli beam to show the closed-loop performance.
\end{abstract}

Keywords: balanced truncation; infinite-dimensional systems; low order controller; model reduction; reciprocal transformation.

\section{Introduction}

The physical system described by partial differential equation has received much attention in recent years, including design control for that system. Examples of these systems are flexible structure, fluid dynamics, robotic, wave, and so on. In implementation, designing control for that system is difficult since it is of infinite state dimension. Modern controller designs, such as $H_{\infty}$ and linear quadratic Gaussian (LQG) techniques, lead to a controller with the same state dimension as the models from which they are designed. Hence, it is important to have low order controller for infinite-dimensional state space. There are two possible alternatives to achieve a low order controller of infinite dimensional system, i.e. model and controllers' reduction. In the first approach,

*Permanent address: Department of Mathematics, Faculty of Science and Technology, Universitas Airlangga, Kampus C Mulyorejo Surabaya, Indonesia.

Received April $13^{\text {th }}, 2009$, Revised September $8^{\text {th }}, 2009$, Accepted for publication November $10^{\text {th }}, 2009$ 
an infinite order of model is reduced and then the low order controller is designed. In the second approach, an infinite order controller is found and then the order of the controller is reduced. In this paper, we will focus on the first approach.

There are many ways to obtain model reduction which have been proposed over the years. Existing methods, such as balanced truncation [1], optimal Hankel norm approximation, singular perturbation approximation (SPA) [2] are often used to reduce model order of finite dimensional linear time-invariant (FDLTI) systems. It is known that the balanced truncation method gives a good model approximation at high frequency, while SPA method is known to be superior at low frequency. Using properties of Hankel operator, several researches have extended the balanced truncation method $[3,4,5]$ to reduce the model order of infinite dimensional case. The recent paper [6] discussed a computational approach of the balanced truncation method for infinite dimensional system via proper orthogonal decomposition (POD).

Balanced truncations method consists of truncating a balanced realization. The balanced realization of infinite dimensional systems is the realization for which the controllability and observability gramians are both equal to some diagonal operator [3]. Suppose $G(s)=C(s I-A)^{-1} B+D$ is a transfer function of infinite dimensional systems with finite rank input and output operator. The $r$-th order balanced truncation of $G$ is the finite dimensional system with the transfer function $G^{r}(s)=C_{1}\left(s I-A_{11}\right)^{-1} B_{1}+D$. If the realization of $G$ is exponentially stable then $D=\lim _{s \rightarrow \infty} G(s)$ exists (see [7, Lemma 7.3.1, p.364]). Since theory of balanced realizations and truncations exists only for exponentially stable systems, then $G(\infty)=G^{r}(\infty)=D$. It is clear that all reduced-order models obtained by truncation of infinite dimensional systems have perfect matching at infinite frequency. Unfortunately, this method yields the greatest approximation error at the low frequency which is undesirable in many applications. It is very interesting to improve model reduction method for infinite dimensional systems with comparable properties as SPA reduction method. It is known that SPA method for FDLTI systems can be analyzed by using properties of a reciprocal system. In the recent papers [8,9], reciprocal transformation has been applied to study properties of infinite dimensional system via their reciprocal systems. Motivated by these works, we propose a method to designing low order controller of infinite dimensional systems via reduced-order model using balanced truncation and reciprocal transformation.

The paper is organized as follows. Section 2 introduces the abstract form of infinite dimensional systems and some notation used in the paper. Reduced order model via balanced truncation of infinite dimensional systems are reviewed in Section 3. Reduced order model using reciprocal transformation and balanced truncation of infinite dimensional systems are derived in Section 4 
which constitutes the main result of this paper. Numerical computation for obtaining a low order controller of infinite dimensional system is proposed in Section 5. The simulation results are presented in Section 6. Finally, concluding remark are given in Section 7.

\section{Preliminaries}

The infinite dimensional systems dynamics can be presented in the following abstract form:

$$
\begin{aligned}
& \dot{x}(t)=A x(t)+B u(t) \\
& y(t)=C x(t)+D u(t)
\end{aligned}
$$

where $A$ is the infinitesimal generator of $C_{0}$-semigroup $T_{a}(t)$ on Hilbert space $\mathrm{Z}$, and operators $B$ and $C$ are finite rank and bounded; $B \in L\left(\mathrm{C}^{m}, Z\right)$, $C \in L\left(Z, \mathrm{C}^{k}\right)$. Here $L(U, Y)$ is the space of bounded linear operator mapping from $U$ to $Y$. In this paper, we will assume the system (1) is exponentially stable means that the operator $A$ generates the exponentially stable $C_{0}$ semigroup $T_{a}(t)$ on $\mathrm{Z}$. The $C_{0}$-semigroup $T_{a}(t)$ on $\mathrm{Z}$ is exponentially stable [7] if there exist positive constants $M$ and $\alpha$ such that $\left\|T_{a}(t)\right\| \leq M e^{-\alpha t}$, for all $t \geq 0$.

We shall denote the state linear system given by $(1)$ as $(A, B, C, D)$ and transfer function given by $G$, with realization $G(s)=C(s I-A)^{-1} B+D$. We omit the " $D$ " term if it is not relevant. The adjoint of operator $A$ is written as $A^{*}$ and domain of $A$ is denoted by $\mathbf{D}(A)$. A symmetric operator $A$ is self-adjoint if $\mathbf{D}\left(A^{*}\right)=\mathbf{D}(A)$. A self-adjoint operator $A$ on the Hilbert space $\mathrm{Z}$ with its inner product $\langle\cdot, \cdot\rangle$ is nonnegative if $\langle A z, z\rangle \geq 0$ for all $z \in \mathbf{D}(A)$ and positive if $\langle A z, z\rangle>0$ for all nonzero $z \in \mathbf{D}(A)$. We will use the notation $A \geq 0$ for nonnegativity of the self-adjoint operator $A$, and $A>0$ for positivity. The resolvent set of $A$ is the set of all complex numbers $\lambda$ for which $(\lambda I-A)^{-1}$ is exists and bounded linear operator on $Z$.

\section{Reduced Order Model via Balanced Truncation}

In this section, we review some result on balanced realization and truncation of infinite dimensional system which has been constructed by $[3,4]$. The construction of the balanced truncation of system $(A, B, C)$ can be obtained by eliminating any states that are difficult to control and to observe based upon the controllability and observability gramians. The controllability and observability gramians are defined by 


$$
L_{B}=\int_{0}^{\infty} T_{a}(t) B B^{*} T_{a}^{*}(t) d t, \quad L_{C}=\int_{0}^{\infty} T_{a}^{*}(t) C^{*} C T_{a}(t) d t
$$

Furthermore, the controllability gramian $L_{B}$ and the observability gramian $L_{C}$ of an exponentially stable system $(A, B, C)$ are the unique solutions of their respective Lyapunov equations:

$$
\begin{aligned}
& A L_{B} z+L_{B} A^{*} z+B B^{*} z=0, \forall z \in \mathbf{D}\left(A^{*}\right) \\
& A^{*} L_{C} z+L_{C} A z+C^{*} C z=0, \forall z \in \mathbf{D}(A) .
\end{aligned}
$$

The procedure of balanced realization of infinite dimensional system is based on the singular value and Schmidt vectors of the Hankel operator of the system. Consider transfer function $G$ is the Laplace transform of $h \in L_{1} \cap L_{2}\left(0, \infty ; \mathrm{C}^{m \times k}\right)$, with realization $G(s)=C(s I-A)^{-1} B$. The Hankel operator $\Gamma_{h}: L_{2}\left(0, \infty ; \mathrm{C}^{m}\right) \rightarrow L_{2}\left(0, \infty ; \mathrm{C}^{k}\right)$ defined by

$$
\left(\Gamma_{h}\right)(t)=\int_{0}^{\infty} h(t+s) u(s) \mathrm{d} s, \quad h(t)=C T_{a}(t) B .
$$

Since $h \in L_{1}\left(0, \infty ; \mathrm{C}^{m \times k}\right)$ implies that $\Gamma_{h}$ is a compact [7, Lemma 8.2.4, p. 399] and so $\Gamma_{h}$ has countable many singular values (square roots of the eigenvalues of $\left.\Gamma_{h}^{*} \Gamma_{h}\right) \sigma_{1} \geq \sigma_{2} \geq \ldots \geq \sigma_{r} \geq \ldots \geq 0$ and these are also the Hankel singular values of $G$. The singular values of $\Gamma_{h}$ are also equal to square roots of the eigenvalues of $L_{B} L_{C}$. The Hankel singular values $\sigma_{i}$ are realization invariant; i.e., they comprise a property of transfer function only and are independent of the realization. The balanced realization has the controllability and observability gramians both equal to $\Sigma=\operatorname{diag}\left(\sigma_{1}, \sigma_{2}, \ldots, \sigma_{r}, \ldots\right)$. Furthermore, the transfer function $\quad G(s)=C(s I-A)^{-1} B$ has a balanced realization $(\tilde{A}, \tilde{B}, \tilde{C})=\left(\Sigma^{-\frac{1}{2}} A \Sigma^{\frac{1}{2}}, \Sigma^{-\frac{1}{2}} B, C \Sigma^{\frac{1}{2}}\right)$ on the state $\ell_{2}$, the space of square summable sequences [3]. In [10], it is shown that an exponentially stable infinite dimensional system with finite rank input and output operators has a nuclear Hankel operator; i.e., its singular values satisfy

$$
\sum_{i=1}^{\infty} \sigma_{i}<\infty
$$

Using the infinite matrix representation for operators on $\ell_{2}$, the balanced system $(\tilde{A}, \tilde{B}, \tilde{C}) \quad$ can be partitioned conformably with $\tilde{L}_{B}=\tilde{L}_{C}=\Sigma=\operatorname{diag}\left(\Sigma_{1}, \Sigma_{2}\right), \quad \Sigma_{1}=\operatorname{diag}\left(\sigma_{1}, \ldots, \sigma_{r}\right), \quad \Sigma_{2}=\operatorname{diag}\left(\sigma_{r+1}, \sigma_{r+2}, \ldots\right)$ $\sigma_{r}>\sigma_{r+1}$ as 


$$
\tilde{A}=\left[\begin{array}{ll}
\tilde{A}_{11} & \tilde{A}_{12} \\
\tilde{A}_{21} & \tilde{A}_{22}
\end{array}\right], \quad \tilde{B}=\left[\begin{array}{c}
\tilde{B}_{1} \\
\tilde{B}_{2}
\end{array}\right], \quad \tilde{C}=\left[\begin{array}{ll}
\tilde{C}_{1} & \tilde{C}_{2}
\end{array}\right] .
$$

The $r$-th order balanced truncation of $(A, B, C)$ are given by the finite dimensional system $\left(\tilde{A}_{11}, \tilde{B}_{1}, \tilde{C}_{1}\right)$. The corresponding reduced order transfer function is $G^{r}$. Note that the reduced system $\left(\tilde{A}_{11}, \tilde{B}_{1}, \tilde{C}_{1}\right)$ is stable and balanced with gramian $\Sigma_{1}$. If system $(A, B, C)$ has output normal realization which is constructed in [4], where $L_{B}=\Sigma^{2}$ and $L_{C}=I$ (the identity operator), then the truncation error can be represented as

$$
\left\|G(s)-G^{r}(s)\right\|_{\infty} \leq 2 \sum_{i=r+1}^{\infty} \sigma_{i} .
$$

By (4), the transfer function of the balanced truncations converge in the $H_{\infty}$ norm.

\section{$4 \quad$ Reduced Order Model Using Reciprocal Transformation}

In this section, we develop reduced order model of infinite dimensional systems via reciprocal transformation. The concept of a reciprocal systems of infinite dimensional systems $(A, B, C, D)$ was introduced in papers $[8,9]$. If zero is in the resolvent set of $A$, i.e. operator $A$ has a bounded inverse, then all the generating operator of the reciprocal systems $A^{-1}, A^{-1} B, C A^{-1}$ are bounded. This motivates the following definition.

Definition 1 Let the system $(A, B, C, D)$ is exponentially stable, such that $A$ has a bounded inverse. Its reciprocal system is the system $(\hat{A}, \hat{B}, \hat{C}, \hat{D})$ where

$$
\hat{A}=A^{-1}, \hat{B}=A^{-1} B, \hat{C}=-C A^{-1}, \hat{D}=D-C A^{-1} B=G(0) .
$$

Let $G$ be transfer function of the systems $(A, B, C, D)$ and suppose $\hat{G}$ is transfer function of the systems $(\hat{A}, \hat{B}, \hat{C}, \hat{D})$. From the above definition, we have

$$
G(s)=-C A^{-1}\left(\frac{1}{s} I-A^{-1}\right)^{-1} A^{-1} B-C A^{-1} B+D=\hat{C}\left(\frac{1}{s} I-\hat{A}\right)^{-1} \hat{B}+\hat{D}=\hat{G}\left(\frac{1}{s}\right) .
$$

Let $H_{\infty}(L(U, Y))$ denote the space of $L(U, Y)$-valued functions of complex variable defined in the open right half-plane which are bounded and analytic in 
the open right half-plane, and $G \in H_{\infty}(L(U, Y))$. From (7), it is clear that $G \in H_{\infty}(L(U, Y))$ if only if $\hat{G} \in H_{\infty}(L(U, Y))$.

Lemma 1 If the system $(A, B, C, D)$ is exponentially stable with its reciprocal system $(\hat{A}, \hat{B}, \hat{C}, \hat{D})$, then their controllability and observability gramians are equal.

Proof. In Lemma 4.1.24 of [7] it was shown that the controllability and observability gramians, $L_{B}, L_{C}$, of the exponentially stable $(A, B, C, D)$ are the unique positive definite solutions to the Lyapunov equations

$$
\begin{aligned}
& \left\langle z,\left(A L_{B}+L_{B} A^{*}+B B^{*}\right) z\right\rangle=0, \forall z \in \mathbf{D}\left(A^{*}\right) \\
& \left\langle z_{1},\left(A^{*} L_{C}+L_{C} A+C^{*} C\right) z_{1}\right\rangle=0, \forall z_{1} \in \mathbf{D}(A) .
\end{aligned}
$$

Letting $z=\left(A^{*}\right)^{-1} x_{1}$ in (8) and $z_{1}=A^{-1} x_{2}$ in (9) where $x_{1}, x_{2}$ is arbitrary elements in $\mathrm{Z}$ gives

$$
\begin{aligned}
& \left\langle x_{1},\left(L_{B}\left(A^{-1}\right)^{*}+A^{-1} L_{B}+A^{-1} B B^{*}\left(A^{-1}\right)^{*}\right) x_{1}\right\rangle=0, \\
& \left\langle x_{2},\left(L_{C} A^{-1}+\left(A^{-1}\right)^{*} L_{C}+\left(A^{-1}\right)^{*} C^{*} C A^{-1}\right) x_{2}\right\rangle=0 .
\end{aligned}
$$

Therefore, from equation (6) we obtain following equations

$$
\begin{aligned}
& \left\langle x_{1},\left(\hat{A} L_{B}+L_{B} \hat{A}^{*}+\hat{B} \hat{B}^{*}\right) x_{1}\right\rangle=0, \\
& \left\langle x_{2},\left(\hat{A}^{*} L_{C}+L_{C} \hat{A}+\hat{C}^{*} \hat{C}\right) x_{2}\right\rangle=0 .
\end{aligned}
$$

This shows that the controllability and observability Lyapunov equations of the systems $(A, B, C, D)$ and its reciprocal systems $(\hat{A}, \hat{B}, \hat{C}, \hat{D})$ have the same solutions.

From the above Lemma, the systems $(A, B, C, D)$ and its reciprocal systems $(\hat{A}, \hat{B}, \hat{C}, \hat{D})$ can be connected via the solutions of the Lyapunov equtions. We can apply it to obtain a balanced realization of $(\hat{A}, \hat{B}, \hat{C}, \hat{D})$ on $\ell_{2}$. It is easy to see that if the realization $(\tilde{A}, \tilde{B}, \tilde{C}, D)=\left(\Sigma^{-\frac{1}{2}} A \Sigma^{\frac{1}{2}}, \Sigma^{-\frac{1}{2}} B, C \Sigma^{\frac{1}{2}}, D\right)$ is balanced with gramian $\Sigma$, then $(\tilde{\hat{A}}, \tilde{\hat{B}}, \tilde{\hat{C}}, \hat{D})=\left(\Sigma^{-\frac{1}{2}} \hat{A} \Sigma^{\frac{1}{2}}, \Sigma^{-\frac{1}{2}} \hat{B}, \hat{C} \Sigma^{\frac{1}{2}}, \hat{D}\right)$ is also balanced with the same gramian. We continue the balanced truncation method to produce a low order model of the balanced realization $(\tilde{\hat{A}}, \tilde{\hat{B}}, \tilde{\hat{C}}, \hat{D})$. We will use the infinite matrix representation for the operators with respect to the standard 
orthonormal basis on $\ell_{2}$. Choose a positive integer $r$ such that $\sigma_{r}>\sigma_{r+1}$ and partition the systems $(\tilde{\hat{A}}, \tilde{\hat{B}}, \tilde{\hat{C}}, \hat{D})$ compatibly with the gramian $\Sigma=\operatorname{diag}\left(\Sigma_{1}, \Sigma_{2}\right) \quad$ where $\quad \Sigma_{1}=\operatorname{diag}\left(\sigma_{1}, \sigma_{2}, \ldots, \sigma_{r}\right), \Sigma_{2}=\operatorname{diag}\left(\sigma_{r+1}, \sigma_{r+2}, \ldots\right)$ become

$$
\tilde{\hat{A}}=\left[\begin{array}{cc}
\tilde{\hat{A}}_{11} & \tilde{\hat{A}}_{12} \\
\tilde{\hat{A}}_{21} & \tilde{\hat{A}}_{22}
\end{array}\right], \quad \tilde{\hat{B}}=\left[\begin{array}{c}
\tilde{\hat{B}}_{1} \\
\tilde{\hat{B}}_{2}
\end{array}\right], \quad \tilde{\hat{C}}=\left[\begin{array}{cc}
\tilde{\hat{C}}_{1} & \tilde{\hat{C}}_{2}
\end{array}\right]
$$

In this paper, we assume that the $\sigma_{i}, i=1,2, \ldots, r$ are all distinct. Since we choose $\sigma_{r}>\sigma_{r+1}$, then we have $\Sigma_{1}>0$. The $r$-th order balanced truncation of the systems $(\tilde{\hat{A}}, \tilde{\hat{B}}, \tilde{\hat{C}}, \hat{D})$ are given by the finite dimensional systems $\left(\tilde{\hat{A}}_{11}, \tilde{\hat{B}}_{1}, \tilde{\hat{C}}_{1}, \hat{D}\right)$. Denote its transfer function by $\tilde{\hat{G}}^{r}$.

Theorem 1 The truncated system $\left(\tilde{\hat{A}}_{11}, \tilde{\hat{B}}_{1}, \tilde{\hat{C}}_{1}, \hat{D}\right)$ is balanced with gramian $\Sigma_{1}$ and asymptotically stable .

Proof. The Lyapunov equations have solutions $\Sigma_{1}$ which correspond to the controllability and observability gramians of the system $\left(\tilde{\hat{A}}_{11}, \tilde{\hat{B}}_{1}, \tilde{\hat{C}}_{1}, \hat{D}\right)$ via (10) and (11), we have

$$
\begin{aligned}
& \tilde{\hat{\hat{A}}}_{11} \Sigma_{1}+\Sigma_{1} \tilde{\hat{\hat{A}}}_{11}^{*}+\tilde{\hat{B}}_{1} \tilde{\hat{B}}_{1}^{*}=0, \\
& \tilde{\hat{A}}_{11}^{*} \Sigma_{1}+\Sigma_{1} \tilde{\hat{A}}_{11}+\tilde{\hat{C}}_{1}^{*} \tilde{\hat{C}}_{1}=0 .
\end{aligned}
$$

Therefore, $\left(\tilde{\hat{A}}_{11}, \tilde{\hat{B}}_{1}, \tilde{\hat{C}}_{1}, \hat{D}\right)$ is balanced with gramian $\Sigma_{1}$.

To prove stability, assume that $\tilde{\hat{A}}_{11} v=\lambda v$, with $\lambda$ be an arbitrary eigenvalues of $\tilde{\hat{A}}_{11}$. Since $\Sigma_{1}>0$ and system $\left(\widetilde{\hat{A}}_{11}, \widetilde{\hat{B}}_{1}, \widetilde{\hat{C}}_{1}, \hat{D}\right)$ is finite dimension, it follows that $\left(\tilde{\hat{\hat{A}}}_{11}, \tilde{\hat{C}}_{1}\right)$ is observable, then $\tilde{\hat{C}}_{1} v \neq 0$. Using the observation Lyapunov equation (13) we have

$$
\left(\lambda^{*}+\lambda\right)\left\|\Sigma_{1}^{1 / 2} v\right\|^{2}=-\left\|\hat{\hat{C}}_{1} v\right\|^{2} .
$$


Hence we obtain $\left(\lambda^{*}+\lambda\right)=2 \operatorname{Re}(\lambda) \leq 0$. If $\operatorname{Re}(\lambda)=0$ then $\tilde{\hat{C}}_{1} v=0$, which is a contradiction. We conclude that $\operatorname{Re}(\lambda)<0$, i.e., the truncated system $\left(\tilde{\hat{A}}_{11}, \tilde{\hat{B}}_{1}, \tilde{\hat{C}}_{1}, \hat{D}\right)$ is asymptotically stable.

The following result shows that a low order model using a reciprocal transformation is a good candidate for a reduced order model for systems $(A, B, C, D)$ and hence for $G$. Now define the reciprocal system $\left(A_{11}, B_{1}, C_{1}, \breve{D}\right)$ of the system $\left(\tilde{\hat{A}}_{11}, \tilde{\hat{B}}_{1}, \tilde{\hat{C}}_{1}, \hat{D}\right)$ by

$$
A_{11}=\tilde{\hat{A}}_{11}^{-1}, B_{1}=\tilde{\hat{A}}_{11}^{-1} \tilde{\hat{B}}_{1}, C_{1}=-\tilde{\hat{C}}_{1} \tilde{\hat{\hat{A}}}_{11}^{-1}, \breve{D}=\hat{D}-\tilde{\hat{C}}_{1} \tilde{\hat{A}}_{11}^{-1} \tilde{\hat{B}}_{1},
$$

assuming that $\tilde{\hat{A}}_{11}$ is invertible.

Note that $\tilde{\hat{G}}^{r}$ is transfer function of $\left(\tilde{\hat{A}}_{11}, \tilde{\hat{B}}_{1}, \tilde{\hat{C}}_{1}, \hat{D}\right)$ with realization

$$
\tilde{\hat{G}}^{r} \underset{s}{\left(\frac{1}{s}\right)}=\tilde{\hat{C}}_{1}\left(\frac{1}{s} I-\tilde{\hat{A}}_{11}\right)^{-1} \tilde{\hat{B}}_{1}+\hat{D} .
$$

Let $\breve{G}^{r}$ be transfer function of the systems $\left(A_{11}, B_{1}, C_{1}, \breve{D}\right)$ with realization

$$
\breve{G}^{r}(s)=C_{1}\left(s I-A_{11}\right)^{-1} B_{1}+\breve{D} .
$$

From (14), the relationship between the transfer function $\tilde{\hat{G}}^{r}$ and the transfer function $\breve{G}^{r}$, is given by

$$
\begin{aligned}
\tilde{\hat{G}}^{r} \underset{s}{\left(\frac{1}{s}\right)} & =\hat{D}-\tilde{\hat{C}} \tilde{\hat{A}}_{11}^{-1} \tilde{\hat{B}}_{1}+\tilde{\hat{C}}_{1}\left[\left(\frac{1}{s} I-\tilde{\hat{A}}_{11}\right)^{-1}+\tilde{\hat{A}}_{11}^{-1}\right] \tilde{\hat{B}}_{1} \\
& =\hat{D}-\tilde{\hat{C}} \tilde{\hat{A}}_{11}^{-1} \tilde{\hat{B}}_{1}-\tilde{\hat{C}}_{1} \tilde{\hat{A}}_{11}^{-1}\left(s I-\tilde{\hat{A}}_{11}^{-1}\right)^{-1} \tilde{\hat{A}}_{11}^{-1} \tilde{\hat{B}}_{1} \\
& =\breve{G}^{r}(s) .
\end{aligned}
$$

The stability and balanced realization of the systems $\left(A_{11}, B_{1}, C_{1}, \breve{D}\right)$ are given in the following theorem.

Theorem 2 If the system $\left(\tilde{\hat{A}}_{11}, \tilde{\hat{B}}_{1}, \tilde{\hat{C}}_{1}, \hat{D}\right)$ is asymptotically stable and balanced with gramian $\Sigma_{1}$ so is the reciprocal system $\left(A_{11}, B_{1}, C_{1}, \breve{D}\right)$ with the same gramian. 
Proof. Since the system $\left(\tilde{\hat{A}}_{11}, \tilde{\hat{B}}_{1}, \tilde{\hat{C}}_{1}, \hat{D}\right)$ is balanced with gramian $\Sigma_{1}$, then $\Sigma_{1}$ satisfy the two Lyapunov equations (12) and (13). Multiplying (12) from the left by $\tilde{\hat{A}}_{11}^{-1}$ and from the right by $\left(\tilde{\hat{A}}_{11}^{-1}\right)^{*}$, and then multiplying (13) from the left by $\left(\tilde{\hat{A}}_{11}^{-1}\right)^{*}$ and from the right by $\tilde{\hat{A}}_{11}^{-1}$, yields

$$
\begin{aligned}
& \Sigma_{1}\left(\tilde{\hat{A}}_{11}^{-1}\right)^{*}+\tilde{\hat{A}}_{11}^{-1} \Sigma_{1}+\left(\tilde{\hat{A}}_{11}^{-1} \tilde{\hat{B}}_{1}\right)\left(\tilde{\hat{A}}_{11}^{-1} \tilde{\hat{B}}_{1}\right)^{*}=0, \\
& \Sigma_{1} \tilde{\hat{A}}_{11}^{-1}+\left(\tilde{\hat{A}}_{11}^{-1}\right)^{*} \Sigma_{1}+\left(\hat{\hat{C}} \tilde{\hat{A}}_{11}^{-1}\right)^{*}\left(\hat{\hat{C}} \tilde{\hat{A}}_{11}^{-1}\right)=0 .
\end{aligned}
$$

Substituting (14) to (16) and (17), we have

$$
\begin{aligned}
& A_{11} \Sigma_{1}+\Sigma_{1} A_{11}^{*}+B_{1} B_{1}^{*}=0, \\
& A_{11}^{*} \Sigma_{1}+\Sigma_{1} A_{11}+C_{1}^{*} C_{1}=0,
\end{aligned}
$$

which implies that the reciprocal system $\left(A_{11}, B_{1}, C_{1}, \breve{D}\right)$ is balanced with gramian $\Sigma_{1}$. Since $\tilde{\hat{A}}_{11}$ is asymptotically stable, then $\operatorname{Re}(\lambda)<0$, with $\lambda$ be any eigenvalue of $\tilde{\hat{A}}_{11}$. Then the eigenvalue of $A_{11}$ is $\frac{1}{\lambda}$ since $A_{11}=\tilde{\hat{A}}_{11}^{-1}$. We hence conclude that $\operatorname{Re}\left(\frac{1}{\lambda}\right)<0$, i.e., the reciprocal system $\left(A_{11}, B_{1}, C_{1}, \breve{D}\right)$ is asymptotically stable.

The next theorem gives the error bounds of reduced order model and convergence in the $H_{\infty}$ norm.

Theorem 3 Assume that $\breve{G}^{r}$ is the transfer function of the r-th reduced order model of the balanced and output normal realizations of $G$. Then we have

$$
\left\|G(s)-\breve{G}^{r}(s)\right\|_{\infty} \leq 2 \sum_{i=r+1}^{\infty} \sigma_{i}
$$

In particular $\left\|G(s)-\breve{G}^{r}(s)\right\|_{\infty} \rightarrow 0$ as $r \rightarrow \infty$.

Proof. We have

$$
\left\|G(s)-\breve{G}^{r}(s)\right\|_{\infty} \leq\left\|G(s)-\hat{G}\left(\frac{1}{s}\right)\right\|_{\infty}+\left\|\hat{G}\left(\frac{1}{s}\right)-\tilde{\hat{G}}^{r}\left(\frac{1}{s}\right)\right\|_{\infty}+\left\|\tilde{\hat{G}}^{r}\left(\frac{1}{s}\right)-\breve{G}^{r}(s)\right\|_{\infty} .
$$

From equations (7) and (15) we have $G(s)=\hat{G}\left(\frac{1}{s}\right)$ and $\tilde{\hat{G}}^{r}\left(\frac{1}{s}\right)=\breve{G}^{r}(s)$, such that we obtain 


$$
\left\|G(s)-\breve{G}^{r}(s)\right\|_{\infty} \leq\left\|\hat{G}\left(\frac{1}{s}\right)-\tilde{\hat{G}}^{r}\left(\frac{1}{s}\right)\right\|_{\infty} .
$$

From Lemma 1, if realization of $G$ have output normal realization, then realization of $\hat{G}$ also have output normal realization. Meanwhile, $\tilde{\hat{G}}^{r}$ is the transfer function of the $r$-th order truncations of the balanced and output normal realizations of $\hat{G}$. Therefore, from equation (5), we conclude that

$$
\left\|G(s)-\breve{G}^{r}(s)\right\|_{\infty} \leq\left\|\hat{G}\left(\frac{1}{s}\right)-\tilde{\hat{G}}^{r}\left(\frac{1}{s}\right)\right\|_{\infty} \leq 2 \sum_{i=r+1}^{\infty} \sigma_{i}
$$

By (4), $\sum_{i=1}^{\infty} \sigma_{i}<\infty$, and so (21) would imply that $\left\|G(s)-\breve{G}^{r}(s)\right\|_{\infty} \rightarrow 0$, as $r \rightarrow \infty$.

An important property of this approach is that the truncated model $\breve{G}^{r}$ is equal to $G$ at zero frequency, since

$$
\begin{aligned}
\breve{G}^{r}(0) & =-C_{1} A_{11}^{-1} B_{1}+\breve{D} \\
& =-\left(-\tilde{\hat{C}} \tilde{\hat{A}}_{11}^{-1}\right)\left(\tilde{\hat{A}}_{11}^{-1}\right)^{-1}\left(\tilde{\hat{A}}_{11}^{-1} \tilde{\hat{B}}_{1}\right)+\hat{D}-\tilde{\hat{C}}_{1} \tilde{\hat{A}}_{11}^{-1} \tilde{\hat{B}}_{1} \\
& =\tilde{\hat{C}}_{1} \tilde{\hat{A}}_{11}^{-1} \tilde{\hat{B}}_{1}+G(0)-\tilde{\hat{C}} \tilde{\hat{A}}_{11}^{-1} \tilde{\hat{B}}_{1} \\
& =G(0),
\end{aligned}
$$

with assumption that zero is in the resolvent set of $A$. It is clear from the discussion above that the reduced order model using reciprocal transformation of infinite dimensional systems gives certain accuracy at low frequency.

\section{Numerical Algorithm}

This section addresses the question of computing the balanced realization and reciprocal transformation followed by low order controller design of infinite dimensional systems based on the proposed method. In the previous section it was assumed that the operator-valued Lyapunov equations can be derived analytically. In general, we cannot obtain an exact solution of the infinite dimensional Lyapunov equations. In this case, a convergent numerical scheme must be utilized as in $[5,11]$. The following algorithm is proposed to find the reduced order model described in the previous section:

1. Find approximating sequence $\left(A^{n}, B^{n}, C^{n}, D^{n}\right)$ to $(A, B, C, D)$, where $n$ is the order of the approximation scheme. 
2. Compute the numerical approximations $L_{B}^{n}$ and $L_{C}^{n}$ to the Lyapunov equations corresponding to $\left(A^{n}, B^{n}, C^{n}, D^{n}\right)$ for sufficiently large $n$.

3. Obtain the balanced realization $\left(\tilde{A}^{n}, \tilde{B}^{n}, \tilde{C}^{n}, D^{n}\right)$ of the systems $\left(A^{n}, B^{n}, C^{n}, D^{n}\right)$ using a similarity transformation.

4. Find reciprocal transformation $\left(\hat{A}^{n}, \hat{B}^{n}, \hat{C}^{n}, \hat{D}^{n}\right)$ of the systems $\left(\tilde{A}^{n}, \tilde{B}^{n}, \tilde{C}^{n}, D^{n}\right)$ as defined in (6).

5. Apply the balanced truncation method to determine the $r$-th order systems $\left(\hat{A}_{11}^{r}, \hat{B}_{1}^{r}, \hat{C}_{1}^{r}, \hat{D}^{r}\right)$ with $r$ as small as possible.

6. From the reduced order model $\left(\hat{A}_{11}^{r}, \hat{B}_{1}^{r}, \hat{C}_{1}^{r}, \hat{D}^{r}\right)$, find its reciprocal transformation $\left(A_{11}^{r}, B_{1}^{r}, C_{1}^{r}, \breve{D}^{r}\right)$ as defined in (14).

7. Then $\left(A_{11}^{r}, B_{1}^{r}, C_{1}^{r}, \breve{D}^{r}\right)$ is the $r$ th-order reduced order model for $\left(A^{n}, B^{n}, C^{n}, D^{n}\right)$ and so for $(A, B, C, D)$.

8. Construct a controller for $\left(A_{11}^{r}, B_{1}^{r}, C_{1}^{r}, \breve{D}^{r}\right)$.

\section{Simulation Result}

In this section, we apply the reduced order model result to design a low order controller for Euler-Bernoulli beam clamped at one end $(\xi=0)$ and free to vibrate at the other end $(\xi=l)$. Let $w(\xi, t)$ represents the deflection of the beam at time $t$ and position $\xi$. The deflection can be controlled by applying a torque $u(t)$ at the clamped end $(\xi=0)$. The external disturbance $v(t)$ induces a uniformly distributed load $\rho d v(t)$. Using linear viscous damping $\left(\gamma_{1}\right)$ and Kelvin-Voigt damping $\left(\gamma_{2}\right)$, the partial differential equation of the beam [11] is given by

$\rho w_{t t}(t, \xi)+\gamma_{1} w_{t}(t, \xi)+E I_{b} w_{\xi \xi \xi \xi}(t, \xi)+\gamma_{2} I_{b} w_{t \xi \xi \xi \xi}(t, \xi)=\frac{\rho \xi}{I_{h}} u(t)+\rho d v(t)$,

with $\rho$ is density of the beam, $E$ is the Young modulus, $I_{b}$ is the moment of inertia, and $I_{h}$ is the hub inertia. The boundary conditions are

$$
\begin{array}{r}
w(t, 0)=w_{\xi}(t, 0)=0, \\
E I_{b} w_{\xi \xi}(t, l)+\gamma_{2} I_{b} w_{t \xi \xi}(t, l)=0, \\
E I_{b} w_{\xi \xi \xi}(t, l)+\gamma_{2} I_{b} w_{t \xi \xi \xi}(t, l)=0,
\end{array}
$$

for $t>0$ and $0<\xi<l$. 
The values of the physical parameters in this example are taken as follows:

Table 1 Physical constants.

\begin{tabular}{cc}
\hline$E$ & $2.1 \times 10^{11} \mathrm{~N} / \mathrm{m}^{2}$ \\
$I_{b}$ & $1.167 \times 10^{-10} \mathrm{~m}^{4}$ \\
$\rho$ & $2.975 \mathrm{~kg} / \mathrm{m}$ \\
$\gamma_{1}$ & $.001 \mathrm{Ns} / \mathrm{m}^{2}$ \\
$\gamma_{2}$ & $.01 \mathrm{Ns} / \mathrm{m}^{2}$ \\
$I_{h}$ & $121.9748 \mathrm{~kg} \mathrm{~m}^{2}$ \\
$d$ & $.041 / \mathrm{kg}^{2}$ \\
\hline
\end{tabular}

Let $H$ be the closed linear subspace of the Sobolev space $H^{2}(0,1)$

$$
H=\left\{w \in H^{2}(0,1): w(0)=w_{\xi}(0)=0\right\}
$$

and define the state-space $Z=H \times L_{2}(0,1)$ with state $x(t)=\left(w(t, \cdot), w_{t}(t, \cdot)\right)$.

If the tip deflection of velocity are measured, a state-space formulation of the above partial differential equation can be presented in the following abstract form

$$
\begin{aligned}
& \dot{x}(t)=A x(t)+B_{1} v(t)+B_{2} u(t) \\
& y(t)=C x(t),
\end{aligned}
$$

where

$$
A=\left[\begin{array}{cc}
0 & I \\
-\frac{E I_{b}}{\rho} \frac{d^{4}}{d \xi^{4}} & -\frac{\gamma_{2} I_{b}}{\rho} \frac{d^{4}}{d \xi^{4}}-\frac{\gamma_{1}}{\rho}
\end{array}\right], B_{1}=\left[\begin{array}{l}
0 \\
d
\end{array}\right], B_{2}=\left[\begin{array}{c}
0 \\
\frac{\xi}{I_{h}}
\end{array}\right], \quad C x(t)=\left[w_{t}(t, l)\right],
$$

with domain

$$
\begin{aligned}
\mathbf{D}(A)= & \left\{(\varphi, \psi) \in Z: \psi \in H \text { and } M=E I_{b} \varphi_{\xi \xi}+\gamma_{2} I_{b} \psi_{\xi \xi} \in H^{2}(0,1)\right. \\
& \text { with } \left.M(l)=M_{\xi}(l)=0\right\} .
\end{aligned}
$$

In order to formulate a full order approximation, we use a Galerkin finite element method with cubic spline [12]. For the finite element method, the spatial interval is divided into 30 elements, with the length of beam to be $l=7$. This corresponds to full order model of size $n=124$. The approximating system (25) is given by

$$
\dot{x}^{n}(t)=A^{n} x^{n}(t)+B_{1}^{n} v^{n}(t)+B_{2}^{n} u^{n}(t)
$$




$$
y^{n}(t)=C^{n} x^{n}(t) .
$$

The purpose of the controller design is to reduce the vibration effects of the disturbance acting on the beam. Such an objective can be achieved by solving a weighted mixed sensitivity $H_{\infty}$ control according to [13]. The block diagram of the mixed sensitivity problem with the inclusion of the weighting function is shown in Figure 1. The performance specification are obtained by designing controller $K(s)$ which satisfy

$$
\left\|\begin{array}{l}
W_{1}(s) T(s) \\
W_{2}(s) S(s)
\end{array}\right\|_{\infty}<1,
$$

where $T(s)$ is the transfer function from the disturbance $v$ to control input $u$, $S(s)$ is transfer function from $v$ to the output $y$ and $W_{1}$ and $W_{2}$ are the two weighting functions to be selected. We take a high-pass filter $W_{1}$ and a low-pass filter $W_{2}$, respectively, as

$$
W_{1}(s)=\frac{190140 s^{2}+4.562 e 004 s+100.4}{s^{2}+2.334 s+9.019}, \quad W_{2}=w_{2} \times w_{2} \times w_{2},
$$

where $w_{2}=\frac{2}{s+a}$ with $a=2 \pi \times 30$.

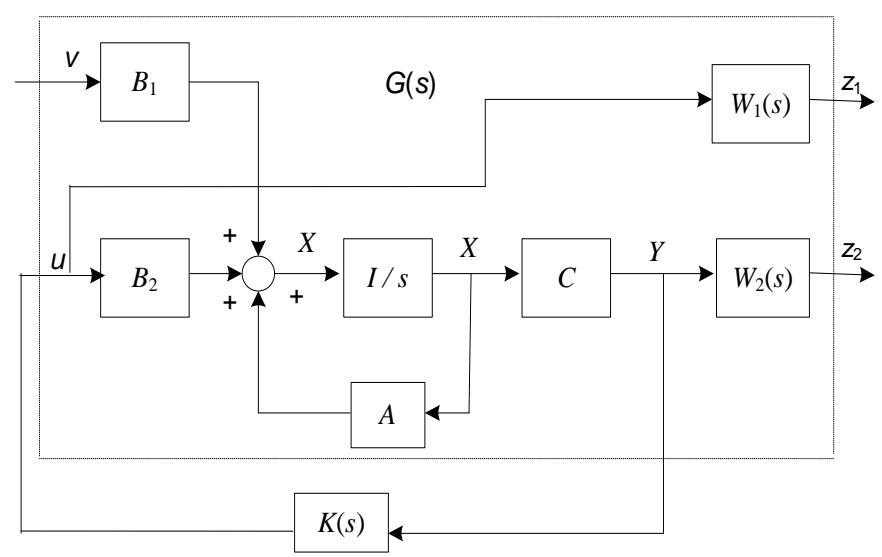

Figure 1 Block diagram of $H_{\infty}$ control.

Furthermore, the order of model is reduced according to the algorithm in section 5. Based on the reduced order model, the 6th-order controllers of the large-scale finite dimensional systems are designed. The time responses of the open-loop and closed-loop system with a temporary step disturbance $v(t)=1$ and a periodic disturbance $v(t)=\sin (\pi t)$ are given in Figure 2 and 3, respectively. 
From these figure, we observe that the 6th-order controllers can attenuate these disturbance.
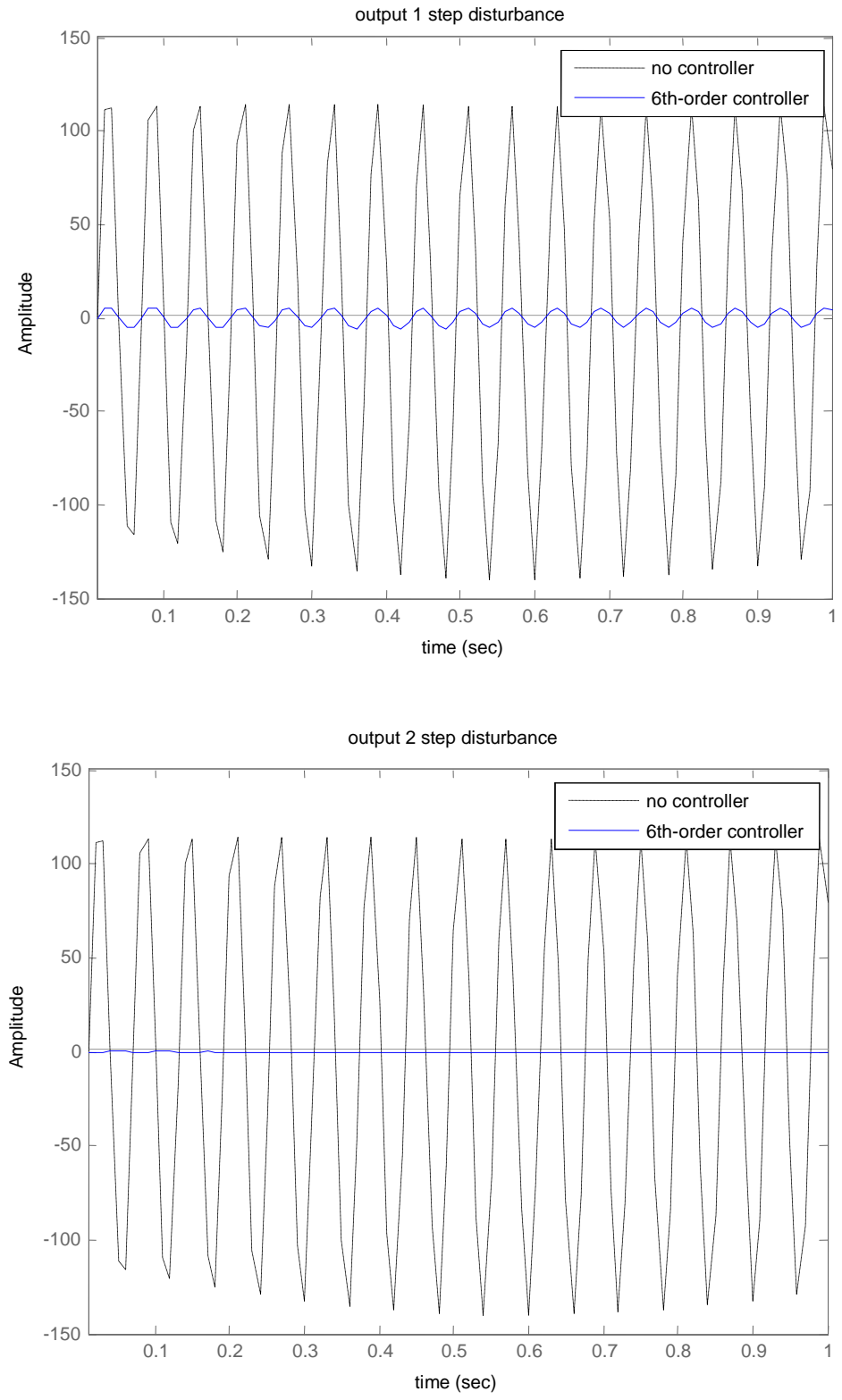

Figure 2 Time response of open and closed-loop to $v(t)=1$. 

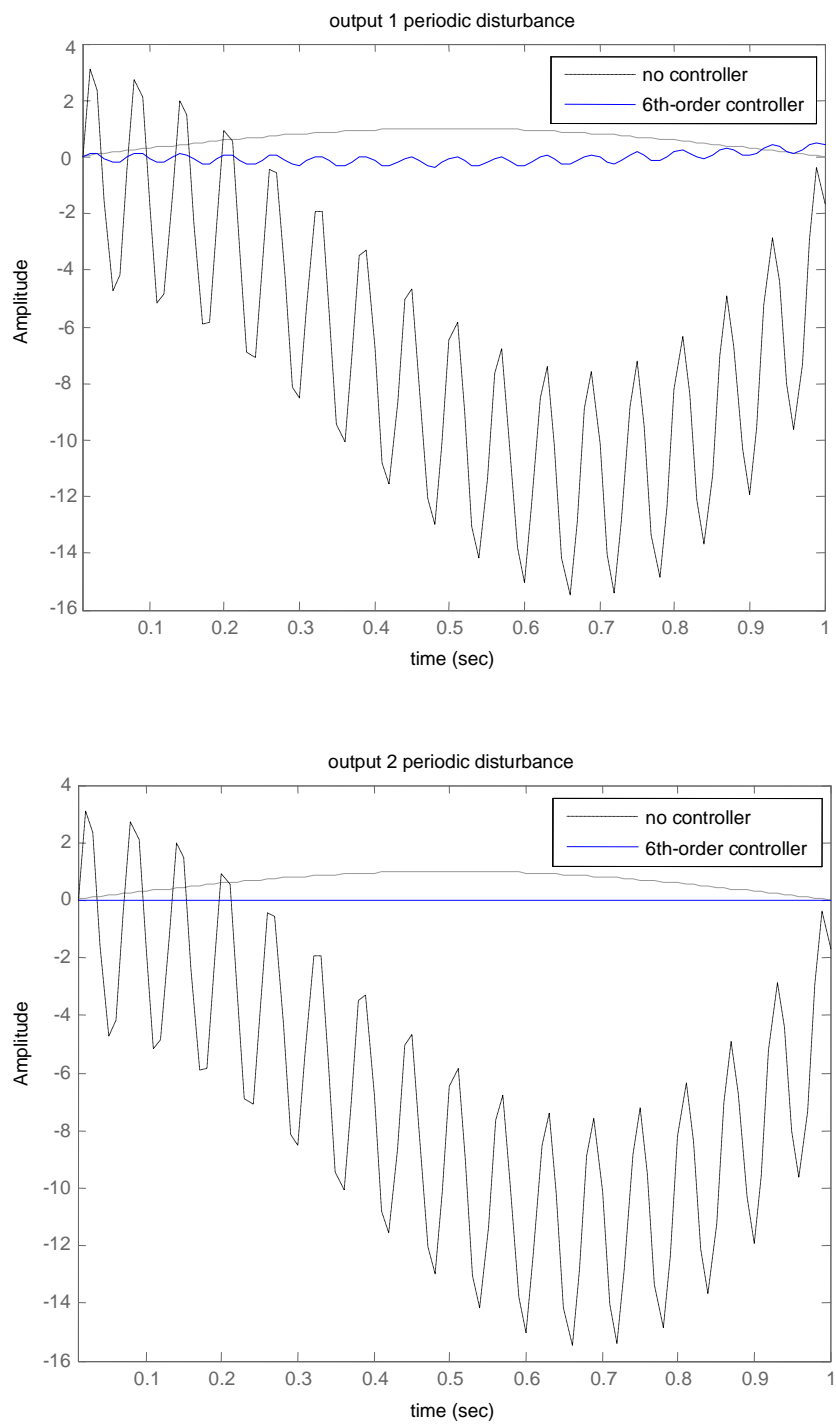

Figure 3 Time response of open and closed-loop to $v(t)=\sin \pi t$.

\section{Concluding Remark}

In this paper, we presented a low order controller design based on reduced order model via reciprocal transformation of infinite dimensional systems. The realization of the reciprocal system is reduced by balanced truncation. This result was further translated using reciprocal transformation as the reduced order model for the original system. The proposed method presented in this paper, preserves the property of singular perturbation approximation (SPA) 
method of finite dimensional system, i.e. its result provide a good reduction errors at low frequencies and therefore it extends [2] for infinite dimensional system. The controller was designed for the reduced order model with the low order controller. The low order controller is applied to reject the disturbance acting on the beam. The simulations showed the effectiveness of the 6 th-order controller to reduce the vibration of the beam.

\section{References}

[1] Moore, B., Principal Component Analysis in Linear Systems: Controllability, Observability, and Model Reduction, IEEE Trans. Automat. Control, AC-26, 17 - 32, 1981.

[2] Liu, Y. \& Anderson, D. O., Singular Perturbation Approximation of Balanced Systems, Int. J. Control, 50, 1379 - 1405, 1989.

[3] Curtain, R.F. \& Glover, K., Balanced Realisation for InfiniteDimensional Systems, in Operator Theory and Systems: Proc. Workshop Amsterdam, 87 - 104, 1986.

[4] Glover, K., Curtain, R.F., \& Partington, J.R., Realisation and Approximation of Linear Infinite-Dimensional Systems with Error Bounds, SIAM J. Control and Optim. 26, 863 - 898, 1989.

[5] Curtain, R.F., Model Reduction for Control Design for Distributed Parameter Systems, in Research Direction in Distributed Parameter Systems, SIAM, Philadelphia, PA, 95 - 121, 2003.

[6] Singler, J.R., \& Batten, B.A., Balanced Proper Orthogonal Decomposition for Model Reduction of Infinite Dimensional Linear Systems, Proceedings of the Int. Conf. on Computational and Mathematical Methods in Science and Engineering, CMMSE, 2007.

[7] Curtain, R.F., \& Zwart, H.J., An Introduction to Infinite-Dimensional Systems, Springer-Verlag, New York, 1995.

[8] Curtain, R.F., Regular Linear Systems and Their Reciprocals: Applications to Riccati Equations, Systems Control Lett, 49, 81 - 89, 2003.

[9] Curtain, R.F., Reciprocals Regular Linear Systems: A Survey, Proceeding MTNS, 2002.

[10] Curtain, R.F. \& Sasane, A.J., Compactness and Nuclearity of the Hankel Operator and Internal Stability of Infinite-Dimensional State Linear Systems, Int. Journal of Control, 74, 1260 - 1270, 2001.

[11] Ito, K. \& Morris, K.A., An Approximation Theory of Solutions to Operator Riccati Equations for $H_{\infty}$ control, SIAM J. Control and Optim. 36, 82 - 99, 1998.

[12] Reddy, J.N., An Introduction to The Finite Element Method, McGrawHill, Singapore, 1984.

[13] Kar, I.N., Miyakura, T. \& Seto, K., Bending and Torsional Vibration Control of A Flexible Plate Structure using $H_{\infty}$-Based Robust Control Law, IEEE Trans. on Control Systems Technology, 8, 545 - 553, 2000. 